23

\title{
Двухстадийный зондовый атомизатор для зеемановской атомно-абсорбционной спектрометрии с высокочастотной модуляцией поляризации излучения
}

\author{
(C) Р.Р. Хайбуллин ${ }^{1}$, Д.С. Ирисов ${ }^{2}$, О.Б. Салихова ${ }^{1}$, Ю.А. Захаров ${ }^{1}$ \\ ${ }^{1}$ Институт фризики Казанского федерального университета, \\ 420008 Казань, Россия \\ 2 „Атзонд“, \\ 420095 Казань, Россия \\ e-mail: rustem21@yandex.ru
}

Поступила в редакцию 20.04.2018 г.

В окончательной редакции 14.09.2018 г.

Предложен двухстадийный зондовый атомизатор для прямого анализа сильно дымящих при испарении проб (нефти, суспензий пищевых продуктов ит.п.) методом зеемановской атомно-абсорбционной спектрометрии с высокочастотной модуляцией поляризации просвечивающего излучения. Обеспечены эффективная вентиляция атомизатора и независимый нагрев вольфрамового зонда в сильном магнитном поле атомизатора для чувствительной регистрации импульсов атомной абсорбции без перекрытия просвечивающего пучка зондом. Работоспособность атомизатора проиллюстрирована на спектрометре МГА-915МД поведением сигналов тестовых элементов $\mathrm{Ag}$ и $\mathrm{Al}$ в зависимости от режимов нагрева, положения зонда и диаметра дозировочного отверстия графитовой печи. Диапазон определяемых концентраций расширен в 50 раз при сохранении погрешности на приемлемом для количественного анализа уровне.

DOI: $10.21883 /$ OS.2019.02.47210.112-18

\section{Введение}

Зеемановская электротермическая атомно-абсорбционная спектрометрия с высокочастотной модуляцией поляризации просвечивающего излучения (ЭТААС-ВЧМП) - один из наиболее чувствительных методов элементного анализа веществ [1,2]. Ее достоинством также является высокое временное разрешение аналитического сигнала, минимизирующее погрешность коррекции быстрых импульсов атомной абсорбции на сопутствующее неселективное поглощение. Эксклюзивным производителем спектрометров такого типа МГА-915, МГА-915МД, МГА-1000 является компания Люмэкс (Санкт-Петербург). Однако их нынешняя конструкция не позволяет осуществлять прямой (без химической подготовки) анализ ряда сложных по матричному составу объектов - сильно дымящихся при атомизации проб пищевых продуктов, горных пород, нефтей и т. п. Дело в том, что графитовую печь для этого метода приходится располагать между полюсами двух плоских постоянных магнитов. Поэтому кожух печи получается в виде объемной коробки с откидывающейся крышкой. Дым заполняет промежуток между печью и крышкой кожуха и затеняет, покрывая копотью, оптический датчик, задающий по обратной связи нагрев печи. В результате температура атомизатора неконтролируемо завышается. Это приводит к недопустимой погрешности измерений и даже к аварийному перегоранию печи.

Графитовые атомизаторы в атомно-абсорбционных спектрометрах (ААC) других оптических систем не используют постоянные магниты, имеют компактный, хорошо вентилируемый кожух и поэтому более приспособлены для атомизации подобных проб [3-5]. Однако такие ААС испытывают трудность с автоматической компенсацией тонко структурированного молекулярного поглощения, которой в принципе нет у ЭТААС-ВЧМП, так как в постоянном магнитном поле в отличие от переменного в других зеемановских ААС не происходит периодического смещения молекулярных полос относительно аналитической линии спектральной лампы, а возникает лишь необходимая селективность атомного пара к ее поляризации. Учитывая то, что разработка приборов для прямого атомно-абсорбционного анализа проб является мировым трендом [6-8], а ЭТААС-ВЧМП имеет важное спектрометрическое преимущество, задача усовершенствования ее атомизатора представляется актуальной. Существенным шагом к ее решению явилось оснащение спектрометра приставкой для зондовой пробоподготовки и атомизации $[9,10]$. Это вместе с дополнительным обдувом аргоном оптического датчика температуры позволило напрямую анализировать на спектрометре МГА-915МД такие сложные пробы, как горные породы и донные отложения. Однако попытки атомизации сырой нефти и суспензий пищевых продуктов, создающих намного больше дыма, оказались неудачными из-за недопустимой дестабилизации температурного режима печи.

Целью настоящей работы является создание атомизатора, позволяющего осуществлять прямой анализ сильно дымящих проб методом ЭТААС-ВЧМП, и оптимиза- 


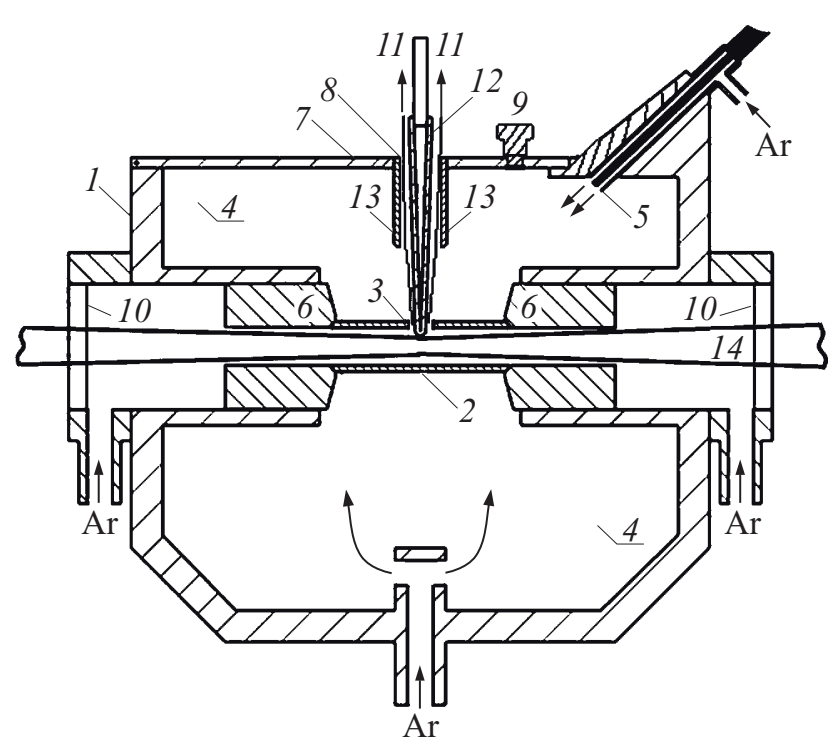

Рис. 1. Двухстадийный зондовый атомизатор в центральном продольном сечении (обозначения см. в тексте).

ция его работы с помощью сигналов абсорбции тестовых элементов $\mathrm{Ag}$ и $\mathrm{Al}$, отличающихся друг от друга летучестью и химической активностью при атомизации.

\section{Экспериментальная часть}

Использовали спектрометр МГА-915МД с автосэмплером и зондовой приставкой Атзонд-1 (Атзонд, Казань). На рис. 1 показан усовершенствованный нами атомизатор. В штатном варианте он состоит из кожуха коробчатого типа 1; графитовой трубчатой печи 2 (длина $28 \mathrm{~mm}$, внутренний диаметр $6 \mathrm{~mm}$ ) с дозировочным отверстием 3; двух плоских магнитов 4, являющихся боковыми стенками кожуха 1 ; световода 5 оптического регулятора температуры печи по свечению ее внешней стенки; графитовых электроконтактов 6; крышки 7 с дозировочным отверстием 8 и ручкой 9 ; кварцевых окон 10. Печь обдувается снаружи $(800 \mathrm{ml} / \mathrm{min})$ и изнутри (250 либо $650 \mathrm{ml} / \mathrm{min}$ ) аргоном (направление подачи схематично показано стрелками, а 11 - это выходящий поток). Усовершенствование включает дополнительный обдув световода 5 аргоном, вольфрамовый зонд 12 и выхлопную трубку 13, выполненную из половинки графитовой печи и ввинченную в отверстие 8 по нарезанной в нем резьбе. Печь просвечивает пучок 14 .

Фото зонда и его держателя показаны в [11]. Зонд изготовлен из вольфрамовой проволоки диаметром $0.9 \mathrm{~mm}$. Такая проволока обладает прочностью, необходимой для выдерживания силы Ампера при пропускании тока через зонд. Зонд нагревали программируемым блоком питания мощностью $450 \mathrm{~W}$ с напряжением $7.5 \mathrm{~V}$. Скорость нагрева регулировали широтно-импульсной модуляцией (ШИМ) этого напряжения с градацией 255 единиц. При значении ШИМ, равном 1, подаются импульсы $7.5 \mathrm{~V}$ длительностью $3.9 \mu \mathrm{s}$ с частотой $1 \mathrm{kHz}$, а при значении 255 - постоянное напряжение $7.5 \mathrm{~V}$. В этом интервале градаций равномерно меняется скважность импульсов напряжения. Также отдельно задавали длительность такого нагрева. Печь ориентировали дозировочным отверстием вверх. Координату кончика зонда отсчитывали вертикально вверх от центра дозировочного отверстия печи, обозначив ее $h_{1}$ для стадии первичной атомизации и $h_{2}$ для вторичной атомизации. Величину ШИМ, $h_{1}$ и $h_{2}$ варьировали при оптимизации сигналов абсорбции. Оптимальные по чувствительности программы двухстадийного зондового атомизатора для водных растворов $\mathrm{Ag}$ и $\mathrm{Al}$ показаны в таблице.

Каплю пробы объемом 10-40 $\mu$ 1 помещают в печь и нагревают по программе (таблица). После сушки и пиролиза печь импульсно нагревают до температуры первичной атомизации. Аргон выносит продукты испарения пробы на кончик зонда, который располагают над дозировочным отверстием 3 печи. Часть продуктов испарения, включая определяемые элементы, фракционно конденсируется на зонде, остальное удаляется через выхлопную трубку, не задерживаясь под крышкой 7. На стадии вторичной атомизации внутренний поток аргона отключают, а зонд погружают в печь и подогревают для быстрого испарения конденсата пробы. Дым при этом образуется в малых количествах и не нарушает работу спектрометра.

Использовали водные азотнокислые растворы Ag и $\mathrm{Al}$ с концентрациями на уровне $0.01-1 \mu \mathrm{g} / \mathrm{ml}$, приготовленные разбавлением стандартного образца Multielement Calibration Standard 3 N9301720 (Perkin-Elmer, CША) $10 \mu \mathrm{g} / \mathrm{ml}$. Добавочной мешающей матрицей служила сырая нефть Республики Татарстан. Фотометрирование осуществляли на линиях ламп с полым катодом $\mathrm{Ag}$ $328.1 \mathrm{~nm}$ и Al $309.3 \mathrm{~nm}$. Для дозирования суспензий заменили конический пластиковый наконечник автосэмплера на стеклянную трубочку внешним диаметром $1.9 \mathrm{~mm}$ и внутренним - $1.2 \mathrm{~mm}$. Увеличенный внутренний диаметр нового наконечника позволял дозировать суспензии с более крупными частицами. Кроме этого, в стаканчик автосэмплера с пробой опускали вибрирующий пестик от оригинального перемешивающего устройства с миниатюрным электромотором. Устройство автоматически включалось за $5 \mathrm{~s}$ до отбора дозы автосэмплером и приводило суспензию в однородное состояние. Это позволяло получать воспроизводимые сигналы от сырой нефти, в которой имеется взвесь.

\section{Результаты и их обсуждение}

\section{Оптимизация условий измерений абсорбционности}

Влияние режима нагрева зонда на абсорбционность. На рис. 2 в качестве примера пунктиром показан график нагрева печи до $2300^{\circ} \mathrm{C}$ и аналитические сигналы алюминия при разной ШИМ. В этом эксперименте 
Оптимальные по чувствительности программы двухстадийного зондового атомизатора для $\mathrm{Ag}$ и $\mathrm{Al}$

\begin{tabular}{|c|c|c|c|c|c|c|}
\hline \multirow{2}{*}{ Шаг } & \multirow{2}{*}{$T$ печи, ${ }^{\circ} \mathrm{C}$} & \multicolumn{2}{|c|}{$\begin{array}{c}\text { Длительность } \\
\text { нагрева печи, s }\end{array}$} & \multirow{2}{*}{$\begin{array}{c}\text { Аргон в печи, } \\
\mathrm{ml} / \mathrm{min}\end{array}$} & \multicolumn{2}{|l|}{ Зонд } \\
\hline & & нагрев & выдержка & & положение, mm & нагрев, ШИМ/s \\
\hline Сушка & 90 & 0.1 & 20 & 270 & Отведен & Выкл. \\
\hline Сушка & 110 & 10 & 10 & 270 & Отведен & Выкл. \\
\hline Пиролиз & 300 & 3.8 & 10 & 270 & Отведен & Выкл. \\
\hline Первичная атомизация & 2400-Al 1900-Ag & 0.8 & 5 & 270 & $h_{1}=3$ & Выкл. \\
\hline Очистка печи & 2700 & 0.2 & 3 & 270 & Отведен & Выкл. \\
\hline Охлаждение & 100 & 5.2 & 16 & 0 & Отведен & Выкл. \\
\hline Вторичная атомизация & 2300-Al 1800-Ag & 1.5 & 6 & 0 & Через $1 \mathrm{~s}$ на $h_{2}=-1.5$ & $100 / 2.4$ \\
\hline Очистка & 2700 & 0.8 & 3 & 270 & $h_{2}=-1.5$ & Выкл. \\
\hline
\end{tabular}

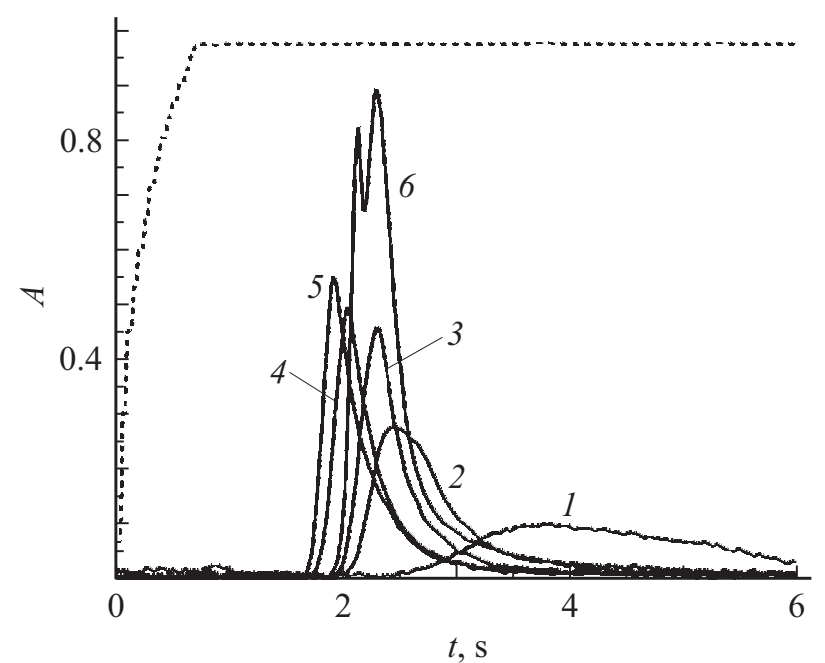

Рис. 2. График нагрева печи до $2300^{\circ} \mathrm{C}$ (пунктир) и аналитические сигналы $20 \mathrm{ng}$ Al (1-4) при разном режиме нагрева зонда ( 1 - ШИМ 45 единиц, длительность $3 \mathrm{~s} ; 2-75$ и $2.8 \mathrm{~s}$; $3-100$ и $2.4 \mathrm{~s} ; 4-125$ и $2.2 \mathrm{~s} ; 5-150$ и $2.1 \mathrm{~s})$ и $60 \mathrm{ng} \mathrm{Al}(6)$ при ШИМ 100 и $2.4 \mathrm{~s}$. Для всех $h_{1}=3 \mathrm{~mm}, h_{2}=-1.5 \mathrm{~mm}$.

$h_{1}=3 \mathrm{~mm}$ и $h_{2}=-1.5 \mathrm{~mm}$. Это обеспечивало максимальное улавливание паров на зонде по данным $[12,13]$ и их последующее испарение в печи без перекрытия зондом просвечивающего пучка [14]. Спектрометр МГА-915МД выдает не оптическую плотность атомного пара, а сигнал пропускания, содержащий инструментальный множитель. В данном случае аналитический сигнал оказался численно близким к оптической плотности пара. Зонд вводили в печь через $1 \mathrm{~s}$ после начала шага атомизации. Видно, что зонд играет роль идеальной платформы Львова, т.е. атомный пар всегда поступает в окончательно прогретую аналитическую зону печи.

Блок питания зонда имел автоматическую защиту от короткого замыкания. Поэтому она всегда срабатывала при подаче напряжения в момент погружения холодного зонда в печь, ограничивая это напряжение. Лишь по мере возрастания электрического сопротивления зонда изза нагрева от печи напряжение достигало заданного с по- мощью ШИМ значения. Без подогрева зонда (ШИМ =0) алюминий не успевает атомизироваться за отведенное время $6 \mathrm{~s}$, и сигнал отсутствует. При ШИМ $=45$ импульс абсорбции от $20 \mathrm{ng} \mathrm{Al}$ низок и затянут (кривая 1 на рис. 2). При увеличении ШИМ сигнал вытягивается вверх (кривые 1-5), но его интегральная величина заметно не изменяется. При ШИМ более 150 единиц сила Ампера ломает зонд, так как он после цикла атомизации становится весьма хрупким и не выдерживает такой изгибной нагрузки. Для исключения риска перегревания зонда длительность включения подогрева сокращали с 3 $\mathrm{s}$ при ШИМ=45 до $2.1 \mathrm{~s}$ при ШИМ = 150 .

Таким образом, установлено, что оптимальным для определения алюминия и подобных ему по летучести элементов является режим нагрева зонда с достаточно широким диапазоном значений - от ШИМ $=150$ при длительности $2.1 \mathrm{~s}$ до ШИМ $=75$ и длительности $2.8 \mathrm{~s}$. Среднеквадратическое отклонение (СКО) аналитического сигнала в данном случае не превышает 6\% при $n=3$. Такой широкий рабочий диапазон параметров удобен, так как не требует тонкой настройки на некий узкий оптимум. В процессе эксплуатации зонда его сопротивление постепенно растет из-за истончения, так как вольфрам окисляется и улетучивается. Однако это обстоятельство практически не влияет на величину интегрального аналитического сигнала. Аналогичная динамика формирования импульсов абсорбции наблюдается и у других элементов. Более летучее серебро начинает быстро атомизироваться уже при ШИМ $=45$ и длительности $3 \mathrm{~s}$ в печи, нагретой до $1800^{\circ} \mathrm{C}$. Усиление параметров нагрева (до алюминиевых) ускоряет диффузионный вынос паров серебра из печи и поэтому слегка уменьшает сигнал абсорбции.

Влияние на абсорбционность температуры первичной атомизации. На рис. 3 дана зависимость нормированных интегральных сигналов абсорбции $\mathrm{Ag}(1)$ и $\mathrm{Al}$ (2) от температуры первичной атомизации водного стандартного раствора. У $\mathrm{Ag}$ сигнал при температурах $<1200^{\circ} \mathrm{C}$ мал из-за того, что часть паров конденсируется на холодных концах печи и не долетает до зонда. При температурах более $2500^{\circ} \mathrm{C}$ сигнал мал из-за 


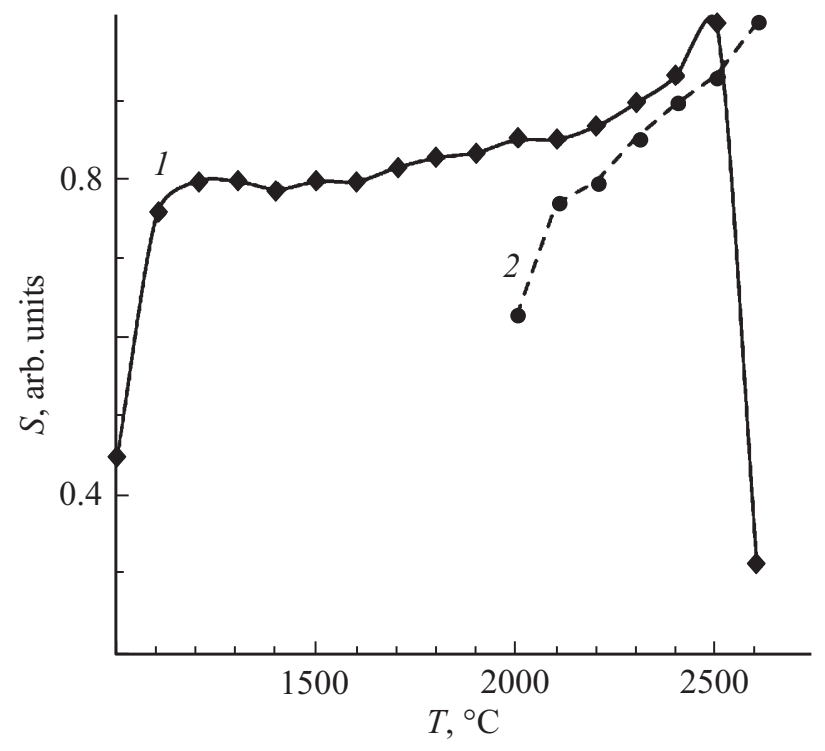

Рис. 3. Зависимость нормированного интегрального сигнала $S$ абсорбции $\mathrm{Ag}-1$ и $\mathrm{Al}-2$ от температуры первичной атомизации. Температура вторичной атомизации $\mathrm{Ag} 1800^{\circ} \mathrm{C}$, Al $2300^{\circ} \mathrm{C}$.

перегрева зонда в потоке защитного газа, в результате чего конденсат преждевременно улетучивается. В этом интервале сигнал плавно возрастает всего лишь на $20 \%$. Это говорит о том, что температура первичной атомизации не требует тонкой настройки и может быть весьма высока, что важно для эффективной диссоциации сложных проб. Аналогичный ход демонстрирует кривая менее летучего Al. Для него температура первичной атомизации должна быть $>2100^{\circ} \mathrm{C}$. Перегрева зонда, приводящего к потерям алюминия, не наблюдается вплоть до максимальной для графитовой печи температуры $2900^{\circ}$ C. Рост сигнала при увеличении температуры в рабочем интервале значений можно объяснить преобразованиями пробы на зонде. В ходе первичной атомизации в продуктах испарения содержится кислород, который оседает вместе с конденсатом пробы на зонде в виде оксидов. При вторичной атомизации этот кислород переносится в печь и препятствует атомизации аналита. При повышении температуры первичной атомизации в потоке паров из печи увеличивается количество оксида и димера углерода. Эти молекулы восстанавливают оксиды металлов, осевшие на зонде с удалением кислорода в составе газообразной двуокиси углерода. Кислорода становится меньше, поэтому эффективность вторичной атомизации $\mathrm{Al}$ возрастает.

Влияние на абсорбционность мешающей небтяной матрицы. Аналогичные эксперименты с Ag и добавками $10 \mu 1$ нефти в качестве мешающей матрицы показали, что аналитические сигналы испытывают двукратное подавление, но остаются достаточно устойчивыми. Неселективное поглощение на стадии вторичной атомизации практически отсутствует. Внутренняя полость кожуха атомизатора, в том числе и крышка, не пачкаются копотью. Видно, что при пиролизе и первичной атомизации формируются мощные потоки дыма, но они эффективно выводятся наружу через выхлопную трубку. Эта трубка к тому же стабилизирует потоки газа и дыма вокруг зонда. Поэтому СКО сигналов не ухудшается, хотя частицы дыма, конкурируя с зондом, улавливают и уносят на себе существенную долю паров аналита. При атомизации нефти в условиях настройки спектрометра на максимальную чувствительность (таблица) обнаружился зашкаливающий сигнал от примеси Al. Для определения его концентрации необходимо понизить чувствительность детектирования.

\section{Расширение диапазона определяемых концентраций}

Градуировочный график в ААС загибается и переходит в насыщение в результате отклонения от закона Ламберта-Бугера-Бэра [15]. При зеемановской коррекции фона этому способствует еще и эффект самообращения абсорбционного сигнала (см. кривую 6 на рис. 2). Поэтому регулировка чувствительности в сторону уменьшения крайне необходима при прямом анализе. Например, в АAC высокого разрешения со сплошным источником спектра [16] для этого фотометрируют крылья линии поглощения. В приборах с электрическим зеемановским корректором фона меняют напряженность магнитного поля. В ЭТААС-ВЧМП пока доступны лишь три классических варианта: 1) переход на менее чувствительную спектральную линию (но не всегда такая имеется), 2) уменьшение дозируемого объема пробы и 3) разбавление пробы. В случае прямого анализа суспензий и твердых навесок последние два приема неприемлемы из-за ухудшения представительности пробы [17].

Известно, что приставка Атзонд-1 позволяет регулировать чувствительность ААС путем изменения $h_{1}$ [12], а также $h_{2}$. В представленном атомизаторе зависимость сигнала $\mathrm{Al}$ от $h_{1}$ при фиксированном $h_{2}=-1.5 \mathrm{~mm}$ тоже имеет экспоненциально спадающий вид, так как при больших $h_{1}$ количество осевшего на зонде пара уменьшается $[13,18]$. Для снижения чувствительности спектрометра в 10 раз нужно увеличить $h_{1}$ с 3 до $20 \mathrm{~mm}$. Дальнейшее увеличение ведет к заметному возрастанию относительного СКО при незначительном изменении величины аналитического сигнала. Например, при $h_{1}=3$ и $h_{2}=-1.5 \mathrm{~mm}$ загиб градуировочного графика начинается при атомизации $20 \mathrm{ng} \mathrm{Al}$, а при $h_{1}=9$ и $h_{2}=-1.5 \mathrm{~mm}$ график линеен до $100 \mathrm{ng}$. У $\mathrm{Ag}$ эти точки графика соответствуют примерно в 6 раз меньшим навескам.

Влияние на абсорбционность положения зонда $h_{2}$ на стадии испарения конденсата. После 150-200 циклов атомизации исходное двухмиллиметровое дозировочное отверстие печи может увеличиться до $3.5 \mathrm{~mm}$ из-за выгорания графита. Это обстоятельство необходимо было учесть, как причину возможного дрейфа 


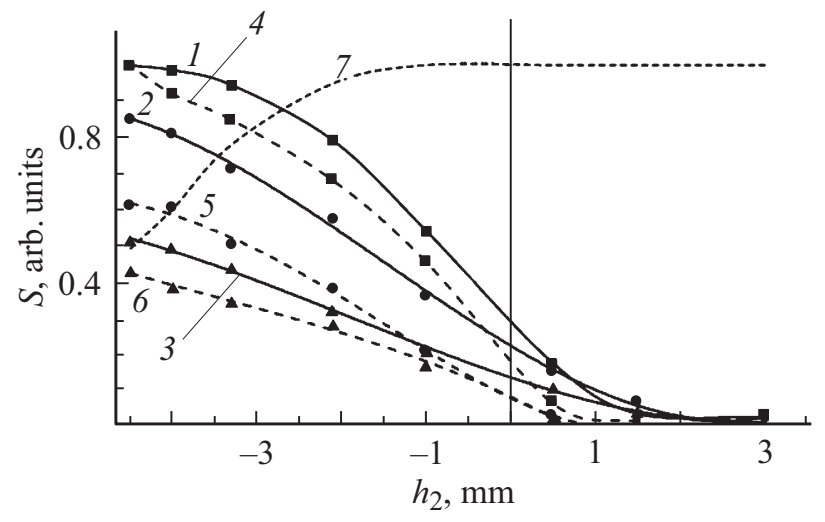

Рис. 4. Нормированные на единицу сигналы $S$ абсорбции $\mathrm{Ag}(1-3), \mathrm{Al}(4-6)$ и энергии просвечивающего пучка 7, полученные с вариацией $h_{2}$ и диаметра дозировочного отверстия печи: $2(1,4) ; 3(2,5)$ и $3.5 \mathrm{~mm}(3,6)$ при $h_{1}=3 \mathrm{~mm}$.

аналитического сигнала. Поэтому на рис. 4 показаны нормированные интегральные сигналы абсорбции $\mathrm{Ag}(1-3)$ и $\mathrm{Al}(4,6)$, полученные при $h_{1}=3 \mathrm{~mm}$ и диаметрах дозировочного отверстия печи 2,3 и $3.5 \mathrm{~mm}$ с вариацией $h_{2}$. Кривой 7 обозначено изменение энергии просвечивающего пучка из-за вводимого в печь зонда. Наибольшие сигналы абсорбции наблюдаются при $h_{2}=-4.5 \mathrm{~mm}$, так как максимальное количество конденсата пробы в этом случае оказывается внутри печи и испаряется в ней. По мере выдвижения зонда из печи сигналы обоих элементов уменьшаются, но в разной степени. На это в первую очередь влияет размер дозировочного отверстия, а также связывание атомного пара примесным кислородом в аргоне и попадающим через дозировочное отверстие из воздуха [19]. Если бы не реакции окисления, то кривые алюминия совпадали бы с кривыми серебра, которые всегда располагаются выше.

Сначала рассмотрим ход кривых 1 и 2 серебра, атомный пар которого в данной ситуации не участвует в химических реакциях. На кривой 1 наблюдаются три участка с разным наклоном. Верхний участок, медленно спадающий на отрезке $h_{2}$ от -4.5 до $-2 \mathrm{~mm}$, объясняется тем, что за пределы печи при единичном перемещении зонда удаляется лишь малая часть сконденсировавшейся на зонде пробы. Второй более крутой участок на отрезке значений $h_{2}$ от -2 до $1 \mathrm{~mm}$ соответствует удалению существенной части конденсата за пределы печи при единичном перемещении зонда. Третий, более пологий участок соответствует ситуации, когда весь конденсат удален из печи, и атомы диффундируют в нее через дозировочное отверстие. Поэтому сигнал слабее уменьшается при единичном перемещении зонда.

Кривая 2 вплоть до отметки $h_{2}=0.5 \mathrm{~mm}$ идет ниже, чем кривая 1 , поскольку атомный пар быстрее покидает печь через увеличенное дозировочное отверстие. При $h_{2}>0.5 \mathrm{~mm}$ весь конденсат оказывается за пределами печи. Приток пара через увеличенное дозировочное отверстие больше, чем через стандартное отверстие. Поэтому кривая 2 при $h_{2}>0.5 \mathrm{~mm}$ идет выше кривой 1 . Увеличение дозировочного отверстия с 2 до $3.5 \mathrm{~mm}$ снижает сигналы Ag и Al примерно в 2 раза. То есть дрейф чувствительности из-за выгорания дозировочного отверстия сравнительно невелик. Он может легко корректироваться через 10-15 рутинных измерений рекалибровкой. В целом рис. 4 показывает, что увеличением $h_{2}$ можно без труда понизить чувствительность на порядок, а одновременным изменением $h_{1}$ и $h_{2}$ - до 50 раз.

По кривой 7 видно, что при погружении в печь глубже, чем на $h_{2}=-1.5 \mathrm{~mm}$ зонд начинает перекрывать просвечивающий пучок. Это обстоятельство, как известно из [14], создает трудности для фотометрирования - снижается светосила спектрометра и усиливается засветка от атомизатора. При $h_{2}=-1.5 \mathrm{~mm}$ влияния зонда на холостой сигнал нет, а чувствительность детектирования атомной абсорбции меньше в 5 раз, чем при традиционной одностадийной атомизации простых водных растворов элементов. Таким образом, если паспортный предел обнаружения в $40 \mu 1$ водного раствора при одностадийной атомизации в МГА-915 МД составляет 3 pg Al [20], то с использованием нашего зонда при $h_{1}=3 \mathrm{~mm}$ и $h_{2}=-1.5 \mathrm{~mm}$ он равен $15 \mathrm{pg}$.

Погружение до $h_{2}=-3 \mathrm{~mm}$ снижает энергию просвечивающего пучка почти в два раза, но примерно так же увеличивает фотометрический шум и сигнал абсорбции. Поэтому концентрационный предел обнаружения не меняется. Исходя из полученных данных, для наиболее чувствительного рутинного анализа проб рекомендуется использовать $h_{2}=(-1.5+0.5) \mathrm{mm}$. Причем этот параметр не требует прецизионной юстировки.

В настоящее время для снижения матричных помех при использовании одностадийной атомизации анализируемые вещества вынуждены растворять и максимально возможно разбавлять перед введением в спектрометр, рассчитывая на его высокую чувствительность. Однако при этом резко возрастают требования к чистоте помещения, химической посуды и реагентов. Представленный в настоящей работе атомизатор меняет существующую концепцию следового анализа. Прямой анализ без разбавления проб позволяет проводить измерения на более высоком уровне концентраций, тем самым снижая затраты лаборатории и риск ошибки.

\section{Выводы}

Разработан и успешно апробирован двухстадийный зондовый атомизатор для прямого микроэлементного анализа сильно дымящих проб (нефти ит.п.) методом электротермической атомно-абсорбционной спектрометрии с высокочастотной модуляцией поляризации просвечивающего излучения. Несмотря на снижение чувствительности измерения в 5 раз по сравнению с одностадийным атомизатором, ее хватает для анализа 
многих объектов, причем с гораздо меньшими затратами и погрешностями за счет исключения химической пробоподготовки. Для проб с повышенным содержанием определяемых элементов атомизатор позволяет снижать чувствительность до 50 крат регулировкой положения зонда. Метрологические характеристики спектрометра с таким атомизатором удовлетворяют требованиям количественного анализа. Таким образом, данный спектрометрический метод полностью пригоден для прямого анализа любых проб, в том числе в виде сложных жидкостей, а также суспензий и твердых навесок.

Авторы признательны руководителям ООО „Атомприбор“ О.В. Евсееву и П.В. Михновцу за техническое содействие и консультации по спектрометрам серии МГА. Работа выполнена при поддержке программы Старт Фонда содействия развитию малых форм предприятий в научно-технической сфере и гранта 063100027 от компании ВР.

\section{Список литературы}

[1] Sholupov S.E., Ganeyev A.A. // Spectrochim. Acta. Part B. 1995. V. 50. P. 1227. doi 10.1016/0584-8547(95)01316-7

[2] Ivanenko N.B., Solovyev N.D., Ivanenko A.A., Ganeev A.A. // Arch. Environ. Contam. Toxicol. 2012. V. 63. P. 299. doi 10.1007/s00244-012-9784-1

[3] Vale M.G.R., Oleszcuk N., dos Santos W.N.L. // Appl. Spectros. Rev. 2006. V. 41. P. 377. doi $10.1080 / 05704920600726167$

[4] Welz B., Vale M.G.R., Borges D.L.G., Heitmann U. // Anal. Bioanal. Chem. 2007. V. 389. P. 2085. doi $10.1007 / \mathrm{s} 00216-$ 007-1555-x

[5] Welz B., Mor'es S., Carasek E., Vale M.G.R., Okruss M., Becker-Ross H. // Appl. Spectr. Rev. 2010. V. 45. P. 327. doi 10.1080/05704928.2010.483669

[6] Borges A.R., Becker E.M., Fran.ois L.L., de Jesus A., Vale M.G.R., Welz B., Dessuy M.B., de Andrade J.B. // Spectrochim. Acta. Part B. 2014. V. 101. P. 213. doi 10.1016/j.sab.2014.08.040

[7] Szymczycha-Madeja A., Welna M., Jedryczko D., Pohl P. // Trends in Analyt. Chem. 2014. V. 55. P. 68. doi 10.1016/j.trac.2013.12.005

[8] Resano M., Flórez M. del R., Queralt I., Marguí E. // Spectrochim. Acta. Part B. 2015. V. 105. P. 38. doi 10.1016/j.sab.2014.09.013

[9] Захаров Ю.А., Окунев Р.В., Хайбуллин Р.Р., Ирисов Д.С., Садыков М.Ф. // Завод. лаб. Диагностика материалов. 2014. T. 80. № 2. С. 12.

[10] Захаров Ю.А., Окунев Р.В., Хасанова С.И., Ирисов Д.С., Хайбуллин Р.P. // Аналитика и контроль. 2013. Т. 17. № 4. C. 414. doi 10.15826/analitika.2013.17.4.006

[11] Захаров Ю.А., Ирисов Д.С., Хайбуллин Р.Р., Чистяков И.В. // Аналитика и контроль. 2015. Т. 19. № 1. С. 32. doi 10.15826/analitika.2015.19.1.008

[12] Захаров Ю.А., Гильмутдинов А.Х., Кокорина О.Б. // Журн. прикл. спектр. 2005. Т. 72. № 1. С. 124. doi 10.1007/s10812-005-0043-3
[13] Захаров Ю.А., Кокорина О.Б., Лысогорский Ю.В., Староверов А.Е. // Журн. аналит. химии. 2012. Т. 67. № 8. С. 790. doi 10.1134/S1061934812060214

[14] Захаров Ю.А., Кокорина О.Б., Окунев Р.В. // Опт. и спектр. 2014. T. 116. № 4. C. 692 . doi 10.7868/S0030403414040291

[15] Гильмутдинов А.Х., Волошин А.В., Нагулин К.Ю. // Успехи химии. 2006. Т. 75. № 4. С. 339. doi 10.1070/RC2006v075n04ABEH002548

[16] Welz B., Becker-Ross H., Florek S., Heitmann U. HighResolution Continuum Source AAS. The Better Way to Do Atomic Absorption Spectrometry. Berlin: Wiley-VCH, 2005. $295 \mathrm{p}$.

[17] Kurfürst U. Solid Sample Analysis: Direct and Slurry Sampling Using GF-AAS and ETV-ICP. Berlin: Springer, 1998. $423 \mathrm{p}$.

[18] Захаров Ю.А., Кокорина О.Б., Лысогорский Ю.В., Севастьянов А.А. // Опт. и спектр. 2008. Т. 105. № 5. С. 744. doi 10.1134/S0030400X08110064

[19] Gilmutdinov A., Chakrabarti C., Hutton J., Mrasov R. // J. Analyt. At. Spectrom. 1992. V. 7. № 7. P. 1047. doi 10.1039/JA9920701047

[20] Спектрометр атомно-абсорбционный МГА-915, МГА915М, МГА-915МД. Руководство по эксплуатации. СПб., 2011. 